\title{
Freqüencia de extrusões de núcleos pulposos cervicais e toracolombares, em cadáveres caninos submetidos à técnica de fenestração
}

\author{
Frequency of cervical e thoracolumbar nucleus pulposus extrusions in cadavers submitted \\ to fenestration technique
}

\author{
Eduardo Alberto Tudury ${ }^{1}$ Maíra Santos Severo² ${ }^{2}$ Maria Juliana Maciel ${ }^{2}$
}

\section{RESUMO}

A fenestração do disco intervertebral faz parte dos procedimentos cirúrgicos indicados para tratamento da doença do disco intervertebral. Este estudo teve como objetivo observar, em 45 cadáveres caninos, se a fenestração ventral e lateral dos discos intervertebrais cervicais e toracolombares respectivamente pode gerar riscos à medula espinhal. Tais cães eram adultos jovens, neurologicamente sadios, com peso oscilando entre sete e $13 \mathrm{~kg}$, dos quais em 25 foi retirado o segmento cervical $\mathrm{C}_{1}-\mathrm{C}_{7}$ e nos demais foi extraído o segmento toracolombar T11-L4, para abertura do canal vertebral (escolheram-se aqueles não afetados por protrusão ou extrusão discal), realização de incisão dorsal do disco até atingir o núcleo pulposo e posterior aplicação e análise das técnicas de fenestração ventral e lateral (ambas com janela e sem janela). Nos 100 discos intervertebrais cervicais fenestrados, observou-se um total de 78 extrusões dorsais de material discal, $55 \%$ delas pertencentes ao grupo da fenestração com janela e $45 \%$ pertencentes ao outro grupo. Dos 120 discos intervertebrais toracolombares fenestrados, observou-se um total de 68 (56,6\%) extrusões de núcleo pulposo, sendo $47 \%$ delas pertencentes ao grupo com janela e $53 \%$ pertencentes ao grupo sem janela. Verificou-se que a fenestração de discos intervertebrais, com ou sem criação de uma janela no ânulo fibroso, pode deslocar núcleo pulposo para o interior do canal vertebral. Foi observada, através de análise estatística, diferença significativa entre os resultados obtidos das fenestrações dos discos intervertebrais cervicais $e$ toracolombares. Porém não ocorreram tais diferenças ao se compararem os métodos de fenestração com e sem janela, realizados tanto na região cervical quanto na toracolombar.

Palavras-chave: coluna vertebral, protrusão de disco, fenestração, cães.

\section{ABSTRACT}

Intervertebral disc fenestration is among the recommended surgical procedures in the management of intervertebral disc disease. It was designated this study, to evaluate the potential for damage to the spinal cord during ventral cervical and lateral thoracolumbar fenestration procedures. Fourty five cadavers of stray dogs of both sexes and various breeds, were used. All dogs were young adults, neurologically normal and with body weight ranging from 7 to $13 \mathrm{~kg}$. From $25 \mathrm{dogs}$, the cervical spinal segment comprehending $C_{1}-C_{7}$ was freshly collected after death, while from the remaining $20 \mathrm{dogs}$, the thoracolumbar segment $T_{11-L 4}$ was equally freshly harvest. The spinal canal was opened (all the dogs were free of intervertebral disc disease) and a dorsal incision was performed through the annulus fibrosus of the intervertebral disc until the nucleus pulposus was reached. A either ventral or lateral fenestration, with and without window openening was immediately performed. From a total of 100 fenestrated cervical intervertebral discs, 78 dorsal extrusions of disc material were observed, being $55 \%$ in the window group and $45 \%$ in the non-windowed group. From the 120 thoracolumbar fenestrated discs, 68 (56.6\%) presented dorsal extrusion of nucleus pulposus material into the spinal canal. Fourty-seven of these occurred in the window group, whereas $53 \%$ of the extrusions occurred in the non-window group. It was verified that intervertebral disc fenestration, with or without the opening of a window in the annulus fibrosus can result in disc material extrusion towards the vertebral canal. The incidence of extrusion was significantly higher in the cervical region when compared to the thoracolumbar area $(p<0.01)$, but there were no differences in disc extrusion rates between the window and non-window techniques in either cervical or thoracolumbar areas.

Key words: spinal surgery, intervertebral disc extrusion, disc fenestration; $\operatorname{dogs}$.

\section{INTRODUÇÃO}

A doença do disco intervertebral, síndrome neurológica comum em cães, é conseqüência da protrusão e da extrusão de material do disco para o

${ }^{1}$ Médico Veterinário, Mestre e Doutor, Professor Adjunto 4, Departamento de Medicina Veterinária (DMV), Universidade Federal Rural de Pernambuco (UFRPE), Rua Dom Manuel de Medeiros s/n, Dois Irmãos, 52171-900, Recife, Pernambuco, Brasil. E-mail: eat-dmv@ufrpe.br

${ }^{2}$ Discentes, Bolsistas do Programa de Iniciação Científica (PIBIC)/Conselho Nacional de Desenvolvimento Tecnológico (CNPq) e Extensão, DMV, UFRPE. 
interior do canal vertebral (TOOMBS \& BAUER, 1993). Neste trabalho, foi adotada a terminologia usada por LeCOUTER \& CHILD (1997), e a afecção foi nomeada como protrusão do disco (projeção do disco dentro do canal vertebral, porém sem ruptura total do ânulo fibroso) e extrusão do disco (deslocamento do núcleo pulposo para dentro do canal vertebral possibilitado pela ruptura completa do ânulo fibroso).

BRAUND (1993) descreveu as seguintes variedades de deslocamentos patológicos do disco intervertebral: a) hérnia dorsal (protrusão) do núcleo pulposo, sem ruptura do ânulo fibroso; b) extrusão dorsal incompleta (sem ruptura do ligamento longitudinal dorsal); c) extrusão dorsal completa com ruptura do ligamento longitudinal dorsal, a qual pode ter direção dorso-mediana, para-mediana, dorsolateral, intraforaminal ou lateral. Já FUNKQUIST (1962) classificou as extrusões toracolombares segundo três tipos: I) o material prolapsado permanece aglomerado acima do disco causando compressão medular localizada ; II) o material do disco encontra-se sobre o ânulo fibroso porém não tão amontoado e menos proeminente; III) o material extravasado acha-se espalhado difusamente craniocaudalmente no espaço epidural.

Na região cervical, ocorrem entre 13,9 a $25,4 \%$ das protrusões de discos intervertebrais dos cães. Dentre os procedimentos cirúrgicos descritos para tratamento da doença do disco cervical em cães, incluem-se a fenestração ventral combinada ou não com fenda em sulco. Para o tratamento da doença do disco toracolombar, tem sido indicada a fenestração lateral junto a procedimentos descompressivos como a laminectomia, a hemilaminectomia e a pediculectomia (COATES, 2000). A fenestração do disco intervertebral remove o material discal remanescente e reduz a recorrência de extrusões, prevenindo futura doença do disco intervertebral (MCKEE, 1992). A cirurgia descompressiva possibilita a remoção do material discal do interior do canal medular, reduzindo a isquemia e a disfunção da medula espinhal provocada pela compressão (COLTER, 1978). Para SCOTT (1997) e ANSELLEM et al. (2003), atualmente é aceito que a cirurgia descompressiva é superior à fenestração em cães paraplégicos, sendo esse o principal objetivo a ser alcançado.

LAHUNTA (1977) definiu a fenestração como a remoção cirúrgica do núcleo pulposo degenerado, e PARKER \& SMALL (1979) como a retirada do núcleo pulposo mediante cuidadosa incisão do ânulo fibroso. Na fenestração com janela, uma abertura em forma de retângulo é feita na parede do disco intervertebral, enquanto na fenestração sem janela uma incisão simples é feita no ânulo fibroso até o núcleo pulposo (DENNY, 1978; HOERLEIN, 1978; CREED \& YTURRASPE, 1998; SEIM 2002). A fenestração realizada através de uma fenda ou janela muito estreita pode gerar deslocamento iatrogênico de núcleo pulposo para o interior do canal vertebral (WHEELER \& SHARP, 1999). Para SCOTT (1997), a fenestração teria mais efeito profilático do que terapêutico, devendo o tratamento se concentrar na descompressão.

$\mathrm{Na}$ cirurgia, a descompressão deve preceder à fenestração, pois se esta última for realizada nas primeiras duas semanas após a aparição dos sinais clínicos, pode romper a cicatriz do ânulo fibroso e deslocar material pulposo para dentro do canal vertebral (PARKER \& SMALL, 1979). JEFFERY (1995) mencionou que a fenestração, realizada nos primeiros dias após a ruptura do ânulo fibroso, pode deslocar mais material em direção à medula espinhal, com conseqüências tão graves como a mielomalácia ascendente. Danos permanentes à propriocepção podem ser atribuídos, em alguns cães, à permanência do material pulposo dentro do canal vertebral. Este material geralmente calcifica por completo (PARKER \& SMALL, 1979) e pode comprimir periodicamente a já deslocada medula espinhal, em determinadas posições da coluna vertebral (BRAUND et al., 1976). Se o material extravasado não for prontamente removido, endurece e adere-se à dura-máter, dificultando sua extirpação (BROWN et al., 1977 e PRATA, 1981).

Este trabalho teve como objetivo verificar se a fenestração (nas alternativas com e sem janela) dos discos intervertebrais toracolombares e cervicais, lateral e ventral respectivamente, pode provocar extrusão de núcleo pulposo para o interior do canal vertebral.

\section{MATERIAL E MÉTODOS}

Foram utilizados cadáveres de 45 cães errantes, sem distinção de sexo ou raça, que foram capturados e submetidos à eutanásia pelo Programa de Controle de Zoonoses da Prefeitura da cidade do Recife. Tais cães eram adultos jovens, neurologicamente sadios e com peso oscilando entre sete e $13 \mathrm{~kg}$. Segmentos vertebrais foram extraídos imediatamente à constatação clínica da morte de tais animais.

Em 25 dos 45 animais estudados, realizouse a extração do segmento cervical da coluna vertebral $\mathrm{C}_{3}-\mathrm{C}_{7}$; enquanto nos demais 20 cães foi extraído o segmento toracolombar $\mathrm{T}_{11}-\mathrm{L}_{4}$. Os segmentos 
espinhais coletados foram submetidos à laminectomia dorsal tipo Funkquist A (SEIM, 2002) e remoção do segmento correspondente da medula espinhal. Verificando-se macroscopicamente nesse instante ausência de protrusão ou extrusão do disco intervertebral, procedeu-se à incisão dorsal de $4 \mathrm{~mm}$ nos ânulos fibrosos, com lâmina de bisturi n ${ }^{\circ} 11^{\text {a }}$, até atingir o núcleo pulposo, para posterior fenestração dos seguintes discos intervertebrais: $\mathrm{C}_{3}-\mathrm{C}_{4} ; \mathrm{C}_{4}-\mathrm{C}_{5} ; \mathrm{C}_{5^{-}}$ $\mathrm{C}_{6} ; \mathrm{C}_{6}-\mathrm{C}_{7}$ e $\mathrm{T}_{11}-\mathrm{T}_{12} ; \mathrm{T}_{12}-\mathrm{T}_{13} ; \mathrm{T}_{13}-\mathrm{L}_{1} ; \mathrm{L}_{1}-\mathrm{L}_{2} ; \mathrm{L}_{2}-\mathrm{L}_{3} ; \mathrm{L}_{3}-\mathrm{L}_{4}$ Foram fénestrados quatro discos intervertebrais cervicais de 25 animais, analisando-se um total de 100 discos, dos quais 50 foram fenestrados com janela e 50 fenestrados sem janela. Em outros 20 cães, foram fenestrados seis discos intervertebrais toracolombares, analisando-se um total de 120 discos. onde em 60 deles realizou-se fenestração com janela, e nos outros 60 , fenestração sem janela; tendo cada disco intervertebral sido fenestrado 10 vezes com cada uma das técnicas adotadas. A fenestração foi realizada mediante incisão do ânulo fibroso, com lâmina de bisturi $\mathrm{n}^{\circ} 11$ montada em cabo $\mathrm{n}^{\circ} 3$, procedendo-se posteriormente à remoção do núcleo pulposo utilizando-se extrator de tártaro SSW n ${ }^{\circ} 3^{\text {b }}$, em forma de arco equivalente a $1 / 4$ de círculo, com espessura de ponta de $0,75 \mathrm{~mm}$ e espessura de base de $1,5 \mathrm{~mm}$.

Após as fenestrações, em cada disco foi observada a ocorrência ou não de extrusão do núcleo pulposo. A confirmação de que a incisão dorsal atingiu o núcleo pulposo foi feita através do mesmo instrumento metálico curvo penetrando pela incisão dorsal e saindo pelo local da fenestração.

As comparações estatísticas da variável referente à extrusão foram realizadas abrangendo os grupos 1-com janela e 2-sem janela, em ambas as regiões coletadas, assim como entre as mesmas, utilizando o teste do qui-quadrado (SNEDECOR \& COCHRAN, 1989) para serem observadas diferenças significativas com relação a cada técnica empregada e entre as frequiências constatadas em cada segmento. Além disso, foi estabelecida a freqüência geral e específica das extrusões em cada tipo de fenestração.

\section{RESULTADOS}

Nos 100 discos intervertebrais cervicais fenestrados, verificaram-se 78 extrusões de material discal, pertencendo $43(55 \%)$ ao grupo da fenestração com janela e 35 (45\%) pertencendo ao outro grupo (Tabela 1).

Nos 120 discos intervertebrais toracolombares fenestrados (Tabela 1), foram observadas $68(56,6 \%)$ extrusões, das quais $32(47 \%)$ ocorreram no grupo da fenestração com janela, e 36 (53\%) no grupo da fenestração sem janela (Tabela 1). Das 32 extrusões do grupo com janela, 40,6\% (13) ocorreram nos discos intervertebrais torácicos (T11T12; T12-T13; T13-L1), e 59,4\% (19) nos discos intervertebrais lombares (L1-L2; L2-L3; L3-L4). Das 36 extrusões do grupo sem janela, 33,3\% (12) ocorreram nos discos intervertebrais torácicos e 66,7\% (24) nos discos intervertebrais lombares.

A forma de prolapso do núcleo pulposo observado neste experimento foi do tipo aglomerado, gelatinoso e dorso-mediano. Pelo teste do quiquadrado, verificou-se diferença altamente significativa $(\mathrm{P}<0,01)$ entre as freqüências de extrusões observadas nas regiões cervical e toracolombar. Entre os métodos de fenestração com e sem janela, realizados tanto nas fenestrações cervicais quanto nas torácicas e lombares, não houve diferença significativa $(\mathrm{P}=0,05)$.

\section{DISCUSSÃO}

Tendo-se verificado extrusão de material pulposo nas fenestrações lateral e ventral dos discos intervertebrais, devido à incisão realizada no ânulo fibroso, concorda-se com a observação de PARKER \& SMALL (1979), JEFFERY (1995) e WHEELER \& SHARP (1999) de que, em pacientes com esta afecção, a fenestração realizada antes de 30 dias (tempo necessário para o ânulo fibroso rompido cicatrizar) poderá empurrar material pulposo para dentro do canal vertebral. Estes achados também tornam-se um alerta, frente à citação de AUTOFAGE et al. (1981) de que a cicatriz que se forma no ânulo fibroso fendido é pequena e pouco resistente.

Enquanto autores como: LEONARD (1971), YTURRASPE \& LUMB (1973), BOJRAB (1975), FLO \& BRINKER (1975), CREED (1976), DENNY (1978), FUNKQUIST (1978), SEEMAN (1980) e DAVIES \& SHARP (1983), trataram pacientes só fenestrando o disco intervertebral sem abordar nem observar o interior do canal vertebral, verificou-se neste estudo a importância da abertura desse canal, com técnicas como laminectomia, hemilaminectomia, pediculectomia ou fenda em sulco, junto à fenestração (COATES, 2000; SEIM, 2002; ANSELLEM, 2003), a fim de se promover a remoção do material já extruso ou que por ventura venha a ser empurrado durante a fenestração (JEFFERY, 1995; WHEELER \& SHARP, 1999), e evitar mediante a fenestração (ação profilática segundo SCOTT, 1997) a recorrência de extrusões de material discal para o interior do canal medular (MCKEE, 1992). Isto é reforçado pelas observações de HOERLEIN (1978, 1979) e PARKER \& SMALL (1979) os quais 
Tabela 1 - Freqüência de extrusões de núcleo pulposo nas fenestrações com janela (FCJ) e sem janela (FSJ) dos discos intervertebrais cervicais e toracolombares.

\begin{tabular}{|c|c|c|c|c|c|c|c|c|}
\hline \multirow{2}{*}{$\begin{array}{l}\text { Segmento } \\
\text { vertebral }\end{array}$} & \multicolumn{2}{|c|}{ Técnica cirúrgica } & \multicolumn{2}{|c|}{ FCJ } & \multicolumn{2}{|c|}{ FSJ } & \multicolumn{2}{|c|}{ Total } \\
\hline & FCJ & FSJ & Com extrusão & Sem extrusão & $\begin{array}{c}\text { Com } \\
\text { extrusão }\end{array}$ & $\begin{array}{c}\text { Sem } \\
\text { extrusão }\end{array}$ & Com extrusão & $\begin{array}{c}\text { Sem } \\
\text { extrusão }\end{array}$ \\
\hline Cervical & 50 & 50 & 43 & 07 & 35 & 15 & 78 & 22 \\
\hline Toracolombar & 60 & 60 & 32 & 28 & 36 & 24 & 68 & 52 \\
\hline
\end{tabular}

explicam que a permanência ou entrada desse material dentro do canal vertebral pode provocar danos permanentes da propriocepção consciente, compressão periódica ou permanente da medula espinhal, e deslocamento mecânico de estruturas nervosas. Ainda, como citado por BROWN et al. (1977), HOERLEIN (1978), PRATA(1981) e SEIM(2002), esse material, quando não é removido, sofre organização fibrosa, cartilaginosa e/ou óssea, aderindo-se à dura-máter dificultando sua extirpação.

A realização de hemilaminectomia ou pediculectomia, técnicas que oferecem adequada abordagem toracolombar para remoção do material prolapsado (McCARTNEY, 1997; COATES, 2000), com menor instabilidade espinhal (SEIM, 2002), junto à fenestração do disco afetado, permitem descomprimir a medula espinhal e evitar recidivas (JEFFERY, 1995; SEIM, 2002). Porém, conforme o sugerido por PARKER \& SMALL (1979), esta descompressão deve preceder à fenestração, pois esta última pode deslocar mais material para o interior do canal vertebral (JEFFERY, 1995; BRUECKER, 1998; WHEELER \& SHARP, 1999), aumentando temporariamente a compressão medular.

Na região cervical, através da fenestração e da fenda em sulco, realiza-se simultaneamente a remoção do núcleo pulposo remanescente e do material já prolapsado para o interior do canal vertebral (JEFFERY, 1995; WHEELER \& SHARP, 1999). Por outro lado, só a fenestração não remove o material prolapsado, podendo oferecer recuperação mais lenta com persistência de dor, ou pior que isso, gerar tetraparesia ou mielomalacia progressiva, pelo deslocamento iatrogênico (confirmado neste trabalho) de material discal para dentro do canal vertebral, causado ou não pela agressividade cirúrgica (JEFFERY, 1995; BRUECKER, 1998; WHEELER \& SHARP, 1999; SEIM, 2002). Apesar do disco C2-C3 ter a maior freqüência de extrusões (COATES, 2000), este espaço não foi estudado, pois foi incisado quando da extração do segmento cervical.
Houve diferença altamente significativa entre as quantidades de extrusões observadas nas regiões cervical e toracolombar, provavelmente devido à manobra realizada na direção ventral para dorsal, que ocorre na fenestração cervical, favorecendo assim a saída de núcleo pulposo para o canal vertebral. Foi surpresa não constatar maior número de extrusões nos grupos fenestração sem janela, nas regiões cervical e toracolombar, pois WHEELER \& SHARP (1999) tinham citado maior possibilidade de deslocamento iatrogênico de material discal, quando a mesma fosse realizada através de janela ou fenda muito estreita.

Só utilizaram-se nesta pesquisa cães cujos discos intervertebrais não apresentaram sinais macroscópicos de protrusão ou extrusão de disco intervertebral, durante a inspeção após a abertura do canal medular, sinais estes relatados por HOERLEIN (1978); BRAUND (1993), LeCOUTER \& CHILD, (1997) e SEIM (2002) como característicos dessas duas afecções. Os prolapsos do tipo I aglomerado e dorsomediano verificados neste experimento, descritos assim por FUNKQUIST (1962) e BRAUND (1993) respectivamente, ocorreram devido à incisão dorsal realizada. A característica gelatinosa decorreu do fato de utilizar-se animais sem raça definida e adultos jovens, pois até os quatro e sete anos respectivamente, somente $25 \%$ e $81 \%$ deles apresentaram degeneração com desidratação do núcleo pulposo (Hansen, apud COATES, 2000).

Os resultados desta pesquisa, apesar de não totalmente extrapoláveis a cães realmente sofredores de degeneração e deslocamentos de discos intervertebrais, sugerem que a fenestração do disco intervertebral nas regiões cervical e toracolombar, em animais cujo anel fibroso dorsal contivesse soluções de continuidade, pode causar a saída de material do disco em direção ao canal vertebral, com conseqüente compressão da medula espinhal. 


\section{CONCLUSÃO}

Observou-se através desta pesquisa, que a fenestração ventral e lateral dos discos intervertebrais cervicais e toracolombares respectivamente, contendo fenda experimentalmente realizada na região dorsal do ânulo fibroso de cadáveres caninos, pode acarretar extrusão de material pulposo para o interior do canal vertebral.

\section{FONTES DE AQUISIÇÃO}

âamina de bisturi $\mathrm{n}^{\mathrm{o}} 11$. Becton Dickinson Ind. Cirúrgicas Ltda., Juiz de Fora - MG

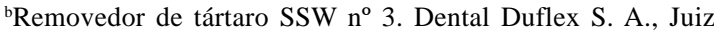
de Fora - MG

\section{REFERÊNCIAS BIBLIOGRÁFICAS}

AMSELLEM, P.M. Loss of deep sensation following thoracolumbar intervertebral disk herniation in dogs. Comp Cont Educ Pract Vet, v.25, n.4, p.266-275, 2003.

AUTOFAGE, A. et al. Élements de traumatologie nervouse. II - La pathologie du disque intervertebral chez le chien: estude clinique. Med Vet, v.132, n.7, p.485-503, 1981

BOJRAB, M.J. Prophylatic thoracolumbar disk fenestration. In: __ Current techniques in small animal surgery. Philadelphia : Lea \& Febiger, 1975. Cap.39, p.404406 .

BRAUND, K.G. et al. Lateral spinal decompression in the dog. J Small Anim Pract, v.17, p.583-592, 1976.

BRAUND, K.G. Intervertebral disk disease. In: BOJRAB M.J. Disease mechanisms in small animal surgery. 2.ed. Philadelphia : Lea \& Febiger, 1993. Cap.129, p.960970

BROWN, N.O.; HELPHREY, M.L.; PRATA, R.G. Thoracolumbar disk disease in the dog: a retrospective analysis of 187 cases. J Am Anim Hosp Assoc, v.13, n.6, p.665-672, 1977.

BRUECKER, K.A. Ventral slot decompression of the herniated cervical disc. In: BOJRAB, M.J. Current techniques in small animal surgery. 4.ed. Baltimore : Williams \& Wilkins, 1998. Cap.46, p.823-826.

COATES, J.R. Intervertebral disk disease. Vet Clin North Am: Small Anim Pract, v.30, n.1, p.77-109, 2000.

COLTER, S.B. Fenestration, decompression, or both? Vet Clin North Am: Small Anim Pract, v.8, n.2, p.379-383, 1978.

CREED, J.E. Thoracolumbar disk syndrome. Fort Collins: Colorado State University, 1976. p.254-263.

CREED, J.E.; YTURRASPE, D.J. Thoracolumbar spine intervertebral disc fenestration. In: BOJRAB, M.J. Current techniques in small animal surgery. 4.ed. Baltimore : Williams \& Wilkins, 1998. Cap.46, p.835-838.
DAVIES, J.V.; SHARP, N.J.H. A comparison of conservative treatment and fenestration for thoracolumbar intervertebral disc disease in the dog. J Small Anim Pract., v.24, p.721$729,1983$.

DENNY, H.R. The lateral fenestration of canine thoracolumbar disc protrusions: a review of 30 cases. J Small Anim Pract, v.19, p.259-266, 1978.

FLO, G.L.; BRINKER, W.O. Lateral fenestration of thoracolumbar disc. J Am Anim Hosp Assoc, v.11, n.5, p.619-626, 1975 .

FUNKQUIST, B. Thoracolumbar disk protrusion with severe cord compression in the dog. I.Clinical and patho-anatomic observations with special reference to the rate of development of the symptoms of motor loss. Acta Vet Scand, n.3, p.256-274, 1962.

FUNKQUIST, B. Investigation of the therapeutic and prophylactic effects of thoracolumbar herniated discs in dogs. Acta Vet Scand, v.19, p.441-457, 1978.

HOERLEIN, B.F. Canine neurology diagnosis and treatment. 3.ed. Philadelphia : Saunders, 1978. 791p.

HOERLEIN, B.F. Comparative disk disease: man and dog. J Am Anim Hosp Assoc, v.15, n.5, p.535-545, 1979.

JEFFERY, N.D. Handbook of small animal spinal surgery. Philadelphia : Saunders, 1995. 236p.

LAHUNTA, A. de. Veterinary neuroanatomy and clinical neurology. Philadelphia : Saunders, 1977. 439p.

LeCOULTER, R.A.; CHILD, G. Afecções da medulla espinal. In: ETTINGER, S.J.; FELDMAN, E.C. Tratado de medicina interna veterinária. 4.ed. São Paulo : Manole, 1997. Cap.83, p.890-980.

LEONARD, E.P. Orthopedic surgery of the dog and cat. 2.ed. Philadelphia : Saunders, 1971. 351p.

McCARTNEY, W. Partial pediculectomy for tha treatment of thoracolombar disc disease. Vet Comparative Orthop Traum, v.10, p.117-121, 1997.

McKEE, W.M. A comparison of hemilaminectomy (with concomitat disc fenestration) and dorsal laminectomy for treatment of thoracolumbar disc protrusion in dogs. Vet Rec, v.130, p.296-300, 1992.

PARKER, A.J.; SMALL, E. The nervous system. In: CATCOTT, E.J. Canine medicine. 4.ed. California : Am Vet Pub, 1979. Cap.12, p.679-812.

PRATA, R.G. Neurosurgical treatment of thoracolumbar disks: the rationale and value of laminectomy with concomitant disk removal. J Am Anim Hosp Assoc, v.17, n.1, p.17-26, 1981 .

SCOTT, H.W. Hemilaminectomy for treatment of thoracolumbar disc disease in the dog: a follow-up study of 40 cases. J Small Anim Pract, v.38, n.11, p.488-494, 1997.

SEEMANN, C.W. Anatomic orientation for lateral thoracolumbar disc fenestration. Vet Med Small Anim Clin, v.75, n.12, p.1865-1868, 1980. 
SEIM, H.B. Neurocirurgia. In: FOSSUM, T.W. Cirurgia de pequenos animais. São Paulo : Roca, 2002. Parte IV, p.1139-1280.

SNEDECOR, G.W.; COCHRAN, W.G. Statistical methods. 8.ed. Iowa : Iowa State University, 1989. 503p.

TOOMBS, J.P.; BAUER, M.S. Intervertebral disc disease. In: SLATTER, D. Textbook of small animal surgery. 2.ed. Philadelphia : Saunders, 1993. p.1070-1087.
WHEELER, S.J.; SHARP, N.J.H. Diagnóstico e tratamento cirúrgico das afecções espinais do cão e do gato. São Paulo : Manole, 1999. 224p.

YTURRASPE, D.J.; LUMB, W.V. A dorsolateral muscle-separating approach for thoracolumbar intervertebral disk fenestration in the dog. J Am Vet Med Assoc, v.162, n.12, p.1037-1040, 1973. 